Алгебра и анализ

Том. 17 (2005), вып. 5
St. Petersburg Math. J.

Vol. 17 (2006), No. 5, Pages 875-895 S 1061-0022(06)00934-4

Article electronically published on July 27, 2006

\title{
SCATTERING BY MAGNETIC FIELDS
}

\author{
D. R. YAFAEV \\ Dedicated to the memory of Ol'ga Aleksandrovna Ladyzhenskaya
}

\begin{abstract}
Consider the scattering amplitude $s\left(\omega, \omega^{\prime} ; \lambda\right), \omega, \omega^{\prime} \in \mathbb{S}^{d-1}, \lambda>0$, corresponding to an arbitrary short-range magnetic field $B(x), x \in \mathbb{R}^{d}$. This is a smooth function of $\omega$ and $\omega^{\prime}$ away from the diagonal $\omega=\omega^{\prime}$, but it may be singular on the diagonal. If $d=2$, then the singular part of the scattering amplitude (for example, in the transversal gauge) is a linear combination of the Dirac $\delta$-function and a singular denominator. Such a structure is typical of the long-range magnetic scattering. This phenomenon is referred to as the long-range Aharonov-Bohm effect. On the contrary, for $d=3$ scattering is essentially of a short-range nature, although, for example, the magnetic potential $A^{(\operatorname{tr})}(x)$ such that $\operatorname{curl} A^{(\operatorname{tr})}(x)=B(x)$ and $\left\langle A^{(\operatorname{tr})}(x), x\right\rangle=0$, decays at infinity as $|x|^{-1}$ only. More precisely, it is shown that, up to the diagonal Dirac function (times an explicit function of $\omega$ ), the scattering amplitude has only a weak singularity in the forward direction $\omega=\omega^{\prime}$. The approach is based on the construction (in the dimension $d=3$ ) of a short-range magnetic potential $A(x)$ corresponding to a given short-range magnetic field $B(x)$.
\end{abstract}

\section{§1. INTRODUCTION}

1.1. In the paper [2] by Aharonov and Bohm (see also [4, 11]), the following mental experiment was discussed. Consider a thin straight solenoid of infinite length, so that the magnetic field $B(x)$ is confined inside this solenoid and is zero outside of it. Consider a beam of particles (electrons) coming from infinity in a certain direction. Suppose that its interaction with the magnetic field inside the solenoid is blocked out by some shield, for example, by a strong repulsive electric field. Nevertheless, the scattering amplitude turns out to be different from zero (the corresponding scattering matrix is not the identity operator). Therefore, it can be expected that, in experiments, a nontrivial interference may be observed behind the solenoid between parts of the initial beam going around the solenoid from the left and from the right. Moreover, this interference picture should depend on the magnetic flux $\Phi$ through a cross section of the solenoid. Of course, this contradicts the classical picture, but conforms perfectly with the principles of quantum mechanics. Indeed, the Schrödinger equation is formulated in terms of a magnetic potential $A(x)$ determined by the equation

$$
\operatorname{curl} A(x)=B(x) \text {. }
$$

In view of translation invariance in the direction of the solenoid, the problem under consideration is two-dimensional. For definiteness, we suppose that the axis of the solenoid coincides with the $x_{3}$-axis, so that $B(x)=(0,0, B(x)), x=\left(x_{1}, x_{2}\right)$, and (1.1) reduces

2000 Mathematics Subject Classification. Primary 47A40, 81U05.

Key words and phrases. Magnetic fields, scattering matrix, gauge transformations, long-range Aharonov-Bohm effect.

(C)2006 American Mathematical Society 
to the equation

$$
\partial A_{2}(x) / \partial x_{1}-\partial A_{1}(x) / \partial x_{2}=B(x)
$$

for the components of the potential $A(x)=\left(A_{1}(x), A_{2}(x), 0\right)$. By the Stokes theorem, the flux $\Phi$ is determined by each of the following two relations:

$$
\Phi=\int_{\mathbb{R}^{2}} B(x) d x=\lim _{R \rightarrow \infty} \int_{|x|=R}\langle A(x), d x\rangle,
$$

where $\langle\cdot, \cdot\rangle$ is the scalar product in $\mathbb{R}^{2}$ or, more generally, in $\mathbb{R}^{d}$. Therefore, $A(x)$ is not zero provided $\Phi \neq 0$. It follows that the scattering is nontrivial even though the magnetic field is zero in the region where particles can penetrate.

Actually, to solve the problem explicitly, Aharonov and Bohm 2 simplified it in the following way. First, they assumed that the solenoid is infinitely thin. Second, instead of an impenetrable shield described mathematically by the Dirichlet boundary condition, they required a regularity of the wave function on the solenoid itself (at $x=0$ ). Thus, strictly speaking, a direct interaction of particles with the magnetic field was not completely excluded in the Aharonov-Bohm (AB) model (this "drawback" was remedied in [11; see also [1). To be more precise, in the paper 2] the Schrödinger operator $H$ with the magnetic potential

$$
A(x)=-\alpha\left(-x_{2}, x_{1}, 0\right)|x|^{-2}, \quad \alpha=-(2 \pi)^{-1} \Phi \in \mathbb{R},
$$

was considered (in accordance with (1.3), in this case the magnetic field equals $-2 \pi \alpha \delta(x)$, where $\delta(x)$ is the Dirac function). For such potentials, the variables in the Schrödinger equation can be separated in the polar coordinates $(r, \theta)$, and for every angular momentum $m=0, \pm 1, \pm 2, \ldots$ the radial equation

$$
-u_{m}^{\prime \prime}+\left((m+\alpha)^{2}-1 / 4\right) r^{-2} u_{m}=\lambda u_{m}
$$

$\left(\lambda>0\right.$ is the energy) can be solved in terms of the Bessel functions $\mathcal{I}_{\nu}$, namely, $u_{m}(r)=$ $r^{1 / 2} \mathcal{I}_{|m+\alpha|}\left(\lambda^{1 / 2} r\right)$. Using the asymptotics of these functions as $r \rightarrow \infty$, we see that the scattering matrix (SM) $S$ for the operator $H$ does not depend on $\lambda$ and has two eigenvalues of infinite multiplicity

$$
s_{m}=e^{i \alpha \pi} \text { for } m \leq-\alpha \text { and } s_{m}=e^{-i \alpha \pi} \text { for } m \geq-\alpha
$$

with the corresponding eigenfunctions $e^{i m \theta}$. Of course, this implies that the SM is nontrivial, that is, $S \neq I$ ( $I$ is the identity operator) if $\alpha \notin 2 \mathbb{Z}$. This fact is known as the $\mathrm{AB}$ effect. It appeared to be surprising, at least from the viewpoint of classical physics, since the magnetic field $B(x)$ is zero for $x \neq 0$. Anyway, the AB effect is a perfect test for the validity of quantum mechanics. Its experimental confirmation was discussed in [7. Note that if we introduce the Planck constant $\hbar$, then $s_{m}(\hbar)=\exp \left(i \alpha \pi \hbar^{-2}\right)$ for $m \leq-\alpha \hbar^{-2}$ and $s_{m}(\hbar)=\exp \left(-i \alpha \pi \hbar^{-2}\right)$ for $m \geq-\alpha \hbar^{-2}$. Therefore, the SM $S(\hbar)$ has no limit as $\hbar \rightarrow 0$, which is consistent with the absence of the $\mathrm{AB}$ effect in classical physics.

Actually, for the $\mathrm{AB}$ potential, not only is the $\mathrm{SM}$ nontrivial, but its properties are typical of long-range scattering. Indeed, the scattering amplitude (the kernel of the SM regarded as an integral operator) equals

$$
s\left(\theta, \theta^{\prime}\right)=(2 \pi)^{-1} \sum_{m=-\infty}^{\infty} s_{m} e^{i m\left(\theta-\theta^{\prime}\right)} .
$$

A simple calculation (see [11]) shows that, for the eigenvalues (1.5), this expression can be written as

$$
s\left(\theta, \theta^{\prime}\right)=\delta\left(\theta-\theta^{\prime}\right) \cos \pi \alpha+i \pi^{-1} e^{-i[\alpha]\left(\theta-\theta^{\prime}\right)} \sin \pi \alpha \text { P.V. }\left(e^{i\left(\theta-\theta^{\prime}\right)}-1\right)^{-1},
$$


where $[\alpha]$ denotes the greatest integer not exceeding $\alpha$. Thus, the scattering amplitude involves a singular denominator (understood in the sense of the principal value) if the magnetic flux $\Phi$ does not belong to $2 \pi \mathbb{Z}$. We use the term "the long-range AharonovBohm effect" for this phenomenon because such a singularity is absent in the case of short-range (both electric and magnetic) potentials.

1.2. We recall that, under natural assumptions, the scattering amplitude $s\left(\omega, \omega^{\prime} ; \lambda\right)$, where $\omega, \omega^{\prime} \in \mathbb{S}^{d-1}$ if $x \in \mathbb{R}^{d}$, is a smooth function away from the diagonal but can be very singular for $\omega=\omega^{\prime}$. This singularity is determined by the decay of the potential at infinity. For short-range potentials satisfying the condition

$$
|A(x)| \leq C(1+|x|)^{-\rho}, \quad \rho>1,
$$

the SM is the sum of the identity operator and an integral operator with a weak diagonal singularity. Moreover, if $\rho \in(1, d)$, then we have the estimate

$$
s\left(\omega, \omega^{\prime} ; \lambda\right)=O\left(\left|\omega-\omega^{\prime}\right|^{-d+\rho}\right), \quad \omega \neq \omega^{\prime}, \omega-\omega^{\prime} \rightarrow 0
$$

(see, e.g., [14]). This singularity is weaker than that of the kernel of a singular integral operator (cf. (1.6)). Moreover, it becomes weaker as long as a potential decays faster at infinity.

For long-range potentials, the diagonal singularity of the scattering amplitude is stronger than in the short-range case, but the diagonal Dirac function disappears. Roughly speaking, for the potentials $A(x)$ asymptotically homogeneous of degree $-\rho, \rho \in(0,1)$, the singular part of the scattering amplitude is given by the formula

$$
s_{0}\left(\omega, \omega^{\prime} ; \lambda\right)=G\left(\omega, \omega-\omega^{\prime} ; \lambda\right) \exp \left(i \Xi\left(\omega, \omega-\omega^{\prime} ; \lambda\right)\right),
$$

where $G$ and $\Xi$ are asymptotically homogeneous functions of $\omega-\omega^{\prime}$ of degrees $-(d-1)\left(1+\rho^{-1}\right) / 2$ and $1-\rho^{-1}$, respectively (see [15]). Thus, the diagonal singularity of the function (1.8) is stronger than that of the kernel of a singular integral operator. Nevertheless, due to oscillations of the second factor in (1.8), the operator with such a kernel is bounded in $L_{2}\left(\mathbb{S}^{d-1}\right)$.

Finally, let us consider the intermediary case of potentials with Coulomb decay $(\rho=1)$ at infinity. Now the results for electric and magnetic potentials are qualitatively different. For electric potentials, the answer is again given by formula (1.8) (see, e.g., 5, 13]), where $G$ is an asymptotically homogeneous function of degree $-d+1$ and $\Xi$ has a logarithmic singularity at $\omega=\omega^{\prime}$. As will be shown below, for arbitrary magnetic potentials satisfying the transversality condition

$$
\langle A(x), x\rangle=0,
$$

the singularity of the scattering amplitude is described by a formula similar to (1.6). However, in this paper we pay special attention to the potentials corresponding to shortrange magnetic fields, for which the answer depends crucially on the dimension of the space.

1.3. Thus, we consider an arbitrary magnetic field satisfying the short-range condition

$$
|B(x)| \leq C(1+|x|)^{-r}, \quad r>2
$$

( $C$ denotes various positive constants whose precise values are of no importance) and study the properties of the corresponding SM. In particular, we find the diagonal singularity of the scattering amplitude. Here our goal is to reveal the difference between the dimensions 2 and 3 .

Of course, when studying the SM for a given magnetic field, we must take into account that, from a theoretical point of view, the SM is determined by a magnetic potential 
$A(x)$ satisfying equation (1.1). In its turn, a solution of this equation is not unique (the gradient of an arbitrary function can be added to $A(x)$ ), which leads to a gauge transformation of the Schrödinger operator. Although the SM are different in different gauges, they are related by a simple formula, i.e., they are covariant with respect to the gauge transformations. This allows us to talk about the SM corresponding to a given magnetic field.

As far as the $\mathrm{AB}$ effect is concerned, the situation is similar in dimensions two and three. For example, consider a toroidal solenoid $\mathbf{T}$ in the space $\mathbb{R}^{3}$. As before, the magnetic field is concentrated inside the solenoid and is zero outside of it. Suppose again that this solenoid is surrounded by a slightly larger toroidal solenoid that excludes a direct interaction of quantum particles with the magnetic field. By the Stokes theorem, the corresponding magnetic potential is nonzero and hence the SM is nontrivial, provided the magnetic flux $\Phi_{s}$ through a transverse crosssection of the solenoid is not zero.

On the contrary, it turns out that the long-range $\mathrm{AB}$ effect always occurs in dimension two (if the total magnetic flux $\Phi$ is not in $2 \pi \mathbb{Z}$ ), but under the assumption (1.10) it cannot occur in dimension three. More precisely, we show that in dimension $d=2$, the diagonal singularity of the scattering amplitude is described by a formula similar to (1.6), i.e., it has a structure typical of the long-range scattering (although the usual wave operators exist in this case). On the contrary, in dimension $d=3$ the structure of the SM is almost the same as for scattering by short-range potentials. Also, we show that condition (1.10) is sharp, that is, the diagonal singularity of the scattering amplitude is described by a formula generalizing (1.6) to the case of $d=3$ if $B(x)$ decays at infinity as a homogeneous function of degree -2 . Note that a condition similar to (1.10) distinguishes also the shortrange electric fields.

A priori, the difference between the dimensions $d=3$ and $d=2$ is not quite obvious. Indeed, for $d=3$ a natural possibility is to choose a potential $A(x)=A^{(\operatorname{tr})}(x)$ satisfying the transversal gauge condition (1.9). Note that this condition is fulfilled (for $x \neq 0$ ) for the $\mathrm{AB}$ potential (1.4). The potential $A^{(\operatorname{tr})}(x)$ decays always as $|x|^{-1}$ at infinity, and hence it can be expected that the SM has a structure typical of the long-range scattering. However, for $d=3$ and a given magnetic field $B(x)$, we can also construct a short-range magnetic potential $A(x)$ satisfying equation (1.1) and condition (1.7). Moreover, if $B(x)$ has compact support, then $A(x)$ is also of compact support. This explains why scattering for $d=3$ is short-range.

The difference between the dimensions $d=3$ and $d=2$ is of a topological nature: if $d \geq 3$, then the set $\mathbb{R}^{d} \backslash\{0\}$ is simply connected, whereas this is not true for $d=2$. No gauge transformation can change the magnetic flux if $d=2$. On the contrary, the absence of a similar invariant for $d=3$ makes the three-dimensional problem essentially more flexible. If $d=2$ and $\Phi \neq 0$, then a magnetic potential cannot even satisfy the condition $A(x)=o\left(|x|^{-1}\right)$ as $|x| \rightarrow \infty$. Indeed, in this case the second integral in (1.3) (the circulation of $A(x)$ over the circle $|x|=R$ ) tends to 0 as $R \rightarrow \infty$, and (1.3) implies that necessarily $\Phi=0$.

As has already been noted, condition (1.10) is sharp; that is, the long-range AB effect is possible even in dimension three if $B(x)$ decays as $|x|^{-2}$. Thus, a long-range behavior of a magnetic field in dimension 3 plays the same role as the topological obstruction in dimension 2 if $\Phi \notin 2 \pi \mathbb{Z}$. Of course, our results for $d=3$ remain true for all dimensions $d \geq 3$ (in the general case $A$ must be viewed as a 1 -form and $B=d A$ as a 2 -form). The electric potentials are assumed to be zero, because they do not add anything new to the phenomena discussed here. In the next section we discuss some elementary facts about pseudodifferential operators (PsDO) acting on a manifold (the unit sphere). Then in $\S 3$ we recall some basic results of scattering theory and discuss the behavior of the 
$\mathrm{SM}$ with respect to gauge transformations. The existence of the long-range $\mathrm{AB}$ effect is established in $\S 4$. On the contrary, in $\S 5$ we prove the absence of this effect for $d=3$ provided condition (1.10) is satisfied.

To a large extent, this paper can be viewed as a survey, although the results of $\S 5$ are, in essence, new. Moreover, compared to [10] and [16, we change the viewpoint, assuming that a magnetic field, rather than a magnetic potential, is given.

\section{§2. Pseudodifferential operators on the Unit SPhere}

2.1. The definition of a PsDO $P$ on the unit sphere $\mathbb{S}^{d-1} \subset \mathbb{R}^{d}$ reduces to that on a domain $\Sigma \subset \mathbb{R}^{d-1}$ (see, e.g., [12]). Roughly speaking, for a neighborhood $\Omega$ of an arbitrary point $\omega_{0} \in \mathbb{S}^{d-1}$ and a diffeomorphism $\varkappa: \Omega \rightarrow \Sigma$, an operator $P: C_{0}^{\infty}(\Omega) \rightarrow$ $C^{\infty}(\Omega)$ reduces by the change of variables $\zeta=\varkappa(\omega)$ to an operator $P_{\varkappa}: C_{0}^{\infty}(\Sigma) \rightarrow$ $C^{\infty}(\Sigma)$. Suppose that, for all $\Omega$ and $\varkappa$, the operators $P_{\varkappa}$ are PsDOs on $\Sigma$, that is,

$$
\left(P_{\varkappa} u\right)(\zeta)=(2 \pi)^{-(d-1) / 2} \int_{\mathbb{R}^{d-1}} e^{i\langle y, \zeta\rangle} p_{\varkappa}(\zeta, y) \hat{u}(y) d y,
$$

where $\hat{u}$ is the Fourier transform of $u$. Then $P$ is called a PsDO on $\mathbb{S}^{d-1}$. We require that the symbols $p_{\varkappa} \in C^{\infty}\left(\Sigma \times \mathbb{R}^{d-1}\right)$ of the PsDO $P_{\varkappa}$ belong to the (Hörmander) class $\mathcal{S}^{m}$ of symbols satisfying the estimates

$$
\left|\partial_{y}^{\alpha} \partial_{\zeta}^{\beta} p_{\varkappa}(\zeta, y)\right| \leq C_{\alpha, \beta}(1+|y|)^{m-|\alpha|}
$$

for all multi-indices $\alpha$ and $\beta$. Then we say that the PsDO $P$ is also of class $\mathcal{S}^{m}$. In terms of the standard PsDO notation, $\zeta$ plays the role of the space variable and the variable $y$ is the dual one.

Actually, it suffices to consider only special diffeomorphisms. For any $\omega_{0} \in \mathbb{S}^{d-1}$, let $\Pi_{\omega_{0}}$ be the hyperplane orthogonal to $\omega_{0}$, and let $\Omega=\Omega\left(\omega_{0}, \gamma\right) \subset \mathbb{S}^{d-1}$ be determined by the condition $\left\langle\omega, \omega_{0}\right\rangle>\gamma>0$. Let $\zeta=\varkappa(\omega)$ be the orthogonal projection of $\omega \in \Omega$ to $\Pi_{\omega_{0}}$; in particular, we assume that $\varkappa\left(\omega_{0}\right)=0$. We denote by $\Sigma$ the orthogonal projection of $\Omega$ to the hyperplane $\Pi_{\omega_{0}}$ and identify the points $\omega \in \Omega$ and $\zeta=\varkappa(\omega)$. The hyperplane $\Pi_{\omega_{0}}$ can be identified with $\mathbb{R}^{d-1}$. Also, we consider the unitary mapping $Z_{\varkappa}: L_{2}(\Omega) \rightarrow L_{2}(\Sigma)$ defined by the formula

$$
\left(Z_{\varkappa} u\right)(\zeta)=\left(1-|\zeta|^{2}\right)^{-1 / 4} u(\omega), \quad \zeta=\varkappa(\omega) .
$$

Note that, compared to the standard definition, for us it is convenient to include the factor $\left(1-|\zeta|^{2}\right)^{-1 / 4}$ in order to make the operator $Z_{\varkappa}$ unitary. We suppose that the operator $P_{\varkappa}=Z_{\varkappa} P Z_{\varkappa}^{*}$ is a PsDO on $\Sigma \subset \mathbb{R}^{d-1}$ with symbol $p_{\varkappa}(\zeta, y)$ of class $\mathcal{S}^{m}=\mathcal{S}^{m}\left(\Sigma \times \mathbb{R}^{d-1}\right)$. The symbol $p_{\varkappa}(\zeta, y)$ is invariant under the diffeomorphisms of $\Sigma$ up to terms of class $\mathcal{S}^{m-1}$. This invariant part, regarded modulo functions in $\mathcal{S}^{m-1}$, is called the principal symbol of the PsDO $P_{\varkappa}$ and will be denoted by $p_{\varkappa}^{(p r)}$. The principal symbol of the PsDO $P$ is well defined (this means that it is independent of the choice of $\varkappa$ ) on the cotangent bundle $T^{*} \mathbb{S}^{d-1}$ of $\mathbb{S}^{d-1}$ by the relation

$$
p(\omega, z)=p_{\varkappa}^{(\mathrm{pr})}(\zeta, y), \quad|\omega|=1, \quad\langle\omega, z\rangle=0,
$$

where $\zeta=\varkappa(\omega)$ and $z={ }^{t} \varkappa^{\prime}(\omega) y$ is the orthogonal projection of $y$ to the hyperplane $\Pi_{\omega}$. Observe also that the kernels $g\left(\omega, \omega^{\prime}\right)$ and $g_{\varkappa}\left(\zeta, \zeta^{\prime}\right)$ of the operators $P$ and $P_{\varkappa}$ regarded as integral operators in $L_{2}(\Omega)$ and $L_{2}(\Sigma)$, respectively, are related by the equation

$$
g\left(\omega, \omega^{\prime}\right)=g_{\varkappa}\left(\zeta, \zeta^{\prime}\right)\left(1-|\zeta|^{2}\right)^{1 / 4}\left(1-\left|\zeta^{\prime}\right|^{2}\right)^{1 / 4}, \quad \omega, \omega^{\prime} \in \Omega .
$$

It is required that $g\left(\omega, \omega^{\prime}\right)$ be a $C^{\infty}$-function away from the diagonal $\omega=\omega^{\prime}$. 
2.2. We need information on the essential spectrum of a PsDO with a homogeneous symbol of order zero. Below, a function $f \in C^{\infty}$ is said to be asymptotically homogeneous of degree $k$ if $f(z)=|z|^{k} f(\hat{z}), \hat{z}=z|z|^{-1}$, for $|z| \geq 1 / 2$. Of course, $1 / 2$ is chosen here for definiteness. Actually, only the behavior of $f(z)$ for large $|z|$ is essential. Let $T_{1}^{*} \mathbb{S}^{d-1} \subset T^{*} \mathbb{S}^{d-1}$ be the set of pairs $(\omega, z)$ such that $\omega, z \in \mathbb{S}^{d-1}$ and $\langle\omega, z\rangle=0$.

Proposition 2.1. Let $P$ be a $\mathrm{PsDO}$ on $\mathbb{S}^{d-1}$ of class $\mathcal{S}^{0}$ with a principal symbol $p(\omega, z)$ asymptotically homogeneous of degree 0 (in the variable $z$ ). Then the essential spectrum $\sigma_{\mathrm{ess}}(P)$ of the operator $P$ in the space $L_{2}\left(\mathbb{S}^{d-1}\right)$ coincides with the range of the function $p(\omega, z)$ restricted to the set $T_{1}^{*} \mathbb{S}^{d-1}$.

As is well known, the kernel $g\left(\omega, \omega^{\prime}\right)$ of a PsDO $P$ regarded as an integral operator can be very singular on the diagonal $\omega=\omega^{\prime}$. Let us find this singularity.

Proposition 2.2. Under the assumptions of Proposition 2.1, the kernel $g\left(\omega, \omega^{\prime}\right)$ of a PsDO $P$ with principal symbol $p(\omega, z)$ admits the representation

$$
g\left(\omega, \omega^{\prime}\right)=p^{(\mathrm{av})}(\omega) \delta\left(\omega, \omega^{\prime}\right)+\text { P.V. } q\left(\omega, \omega^{\prime}-\omega\right),
$$

up to terms of order $O\left(\left|\omega-\omega^{\prime}\right|^{-d+1+\nu}\right)$ for any $\nu<1$ if $d=2$ and for $\nu=1$ if $d \geq 3$. Here $\delta\left(\omega, \omega^{\prime}\right)$ is the Dirac function on the unit sphere,

$$
\begin{aligned}
p^{(\mathrm{av})}(\omega) & =\left|\mathbb{S}^{d-2}\right|^{-1} \int_{\mathbb{S}_{\omega}^{d-2}} p(\omega, \psi) d \psi, \quad \mathbb{S}_{\omega}^{d-2}=\mathbb{S}^{d-1} \cap \Pi_{\omega}, \\
q(\omega, \tau) & =(2 \pi i)^{-d+1}(d-2) ! \int_{\mathbb{S}_{\omega}^{d-2}}\left(p(\omega, \psi)-p^{(\mathrm{av})}(\omega)\right)(\langle\psi, \tau\rangle-i 0)^{-d+1} d \psi,
\end{aligned}
$$

so that, in particular,

$$
\int_{\mathbb{S}_{\omega}^{d-2}} q(\omega, \varphi) d \varphi=0
$$

for all $\omega \in \mathbb{S}^{d-1}$.

Note that the function $q\left(\omega, \omega^{\prime}-\omega\right)$ in (2.1) is homogeneous of degree $-d+1$ in the variable $\omega^{\prime}-\omega$. Therefore, by condition (2.2), the integral operator with this kernel is well defined (as a bounded operator on $L_{2}\left(\mathbb{S}^{d-1}\right)$ ) in the sense of the principal value. Thus, up to an integral operator with a weak diagonal singularity, $P$ is the sum of the operator $P_{0}$ of multiplication by $p^{(\mathrm{av})}(\omega)$ and a singular integral operator. To be explicit,

$$
\left(P_{0} f\right)(\omega)=p^{(\mathrm{av})}(\omega) f(\omega)+\lim _{\varepsilon \rightarrow 0} \int_{\left|\omega^{\prime}-\omega\right|>\varepsilon} q\left(\omega, \omega^{\prime}-\omega\right) f\left(\omega^{\prime}\right) d \omega^{\prime} .
$$

We emphasize that a PsDO $P$ of order zero is determined by its principal symbol only up to terms of class $\mathcal{S}^{-1}$. These latter operators are compact, so that, by the Weyl theorem, the essential spectra of all such PsDOs $P$ are the same. Similarly, the singular parts of the kernels of all such operators $P$ are given by the same formula (2.1), and all remainders are $O\left(\left|\omega-\omega^{\prime}\right|^{-d+1+\nu}\right)$.

Remark 2.3. Let $d=2$. Then $T_{1}^{*} \mathbb{S}$ consists of the points $\left(\omega, \omega^{(+)}\right)$and $\left(\omega, \omega^{(-)}\right)$, where $\omega \in \mathbb{S}$ is arbitrary and $\omega^{(+)}$and $\omega^{(-)}=-\omega^{(+)}$are obtained from $\omega$ by rotation by an angle of $\pi / 2$ and $-\pi / 2$ in the positive (counterclockwise) direction. The integral over $\mathbb{S}_{\omega}^{0}$ reduces to the sum over the points $\omega^{(+)}$and $\omega^{(-)}$, and

$$
\left\langle\omega^{( \pm)}, \omega^{\prime}-\omega\right\rangle= \pm\left|\omega^{\prime}-\omega\right| \operatorname{sgn}\left\{\omega, \omega^{\prime}\right\}+O\left(\left|\omega^{\prime}-\omega\right|^{2}\right), \quad \omega^{\prime} \rightarrow \omega,
$$


where $\left\{\omega, \omega^{\prime}\right\}$ is the oriented angle between the initial vector $\omega$ and the final vector $\omega^{\prime}$. We set

$$
\left\{\begin{aligned}
p^{(\mathrm{av})}(\omega) & =2^{-1}\left(p\left(\omega, \omega^{(+)}\right)+p\left(\omega, \omega^{(-)}\right)\right), \\
p^{(s)}(\omega) & =(2 \pi i)^{-1}\left(p\left(\omega, \omega^{(+)}\right)-p\left(\omega, \omega^{(-)}\right)\right) .
\end{aligned}\right.
$$

Then formula (2.1) for the singular part of the kernel of $P$ can be written in the form

$$
g\left(\omega, \omega^{\prime}\right)=p^{(\text {av })}(\omega) \delta\left(\omega, \omega^{\prime}\right)+p^{(s)}(\omega) \text { P.V. }\left|\omega^{\prime}-\omega\right|^{-1} \operatorname{sgn}\left\{\omega, \omega^{\prime}\right\} .
$$

The proofs of Propositions 2.1 and 2.2 can be found in [16].

\section{§3. SCATTERING MATRIX}

3.1. We briefly discuss some basic facts of scattering theory. Consider the Schrödinger operator

$$
H=(i \nabla+A(x))^{2}, \quad x \in \mathbb{R}^{d}, \quad d \geq 2,
$$

with a real magnetic potential $A(x)=\left(A_{1}(x), \ldots, A_{d}(x)\right)$ satisfying condition (1.7). The dimension $d$ is arbitrary in this section. We do not assume that the function $A(x)$ is differentiable, so that, strictly speaking, $H$ is well defined as a selfadjoint operator in the space $L_{2}\left(\mathbb{R}^{d}\right)$ in terms of the corresponding quadratic form. In general, equation (1.1) should be understood in the sense of distributions.

Let $H_{0}=-\Delta$ be the "free" operator. Under the assumption (1.7), the wave operators

$$
W_{ \pm}=W_{ \pm}\left(H, H_{0}\right)=\underset{t \rightarrow \pm \infty}{s-\lim _{t \rightarrow \infty}} e^{i H t} e^{-i H_{0} t}
$$

exist, are unitary, and possess the intertwining property

$$
H W_{ \pm}=W_{ \pm} H_{0} .
$$

The scattering operator $\mathbf{S}=W_{+}^{*} W_{-}$is unitary and commutes with $H_{0}$. Let $F: L_{2}\left(\mathbb{R}^{d}\right) \rightarrow$ $L_{2}\left(\mathbb{R}_{+} ; L_{2}\left(\mathbb{S}^{d-1}\right)\right)$ be the unitary operator defined by the formula

$$
(F f)(\lambda ; \omega)=2^{-1 / 2} \lambda^{(d-2) / 4} \hat{f}\left(\lambda^{1 / 2} \omega\right), \quad \lambda>0, \quad \omega \in \mathbb{S}^{d-1},
$$

where $\hat{f}=\mathcal{F} f$ is the Fourier transform of $f \in L_{2}\left(\mathbb{R}^{d}\right)$. Clearly, $\left(F H_{0} f\right)(\lambda)=\lambda(F f)(\lambda)$. Since $\mathbf{S} H_{0}=H_{0} \mathbf{S}$, we have

$$
(F \mathbf{S} f)(\lambda)=S(\lambda)(F f)(\lambda),
$$

where the unitary operator $S(\lambda): L_{2}\left(\mathbb{S}^{d-1}\right) \rightarrow L_{2}\left(\mathbb{S}^{d-1}\right)$ is known as the scattering matrix (SM). The scattering amplitude $s\left(\omega, \omega^{\prime} ; \lambda\right), \omega, \omega^{\prime} \in \mathbb{S}^{d-1}$, is the kernel of $S(\lambda)$ regarded as an integral operator. The following assertion is well known (see, e.g., 14, 16]).

Proposition 3.1. Suppose condition (1.7) is fulfilled. Then the operator $T(\lambda)=S(\lambda)-I$ is compact, and it belongs to the trace class if $\rho>d$. If $\rho>d+n, n=0,1,2, \ldots$, then $T(\lambda)$ is an integral operator with kernel of class $C^{n}\left(\mathbb{S}^{d-1} \times \mathbb{S}^{d-1}\right)$.

If

$$
\left|\partial^{\alpha} A(x)\right| \leq C_{\alpha}(1+|x|)^{-\rho-|\alpha|}
$$

for some $\rho \in(1, d)$ and all multi-indices $\alpha$, then the operator $T(\lambda)$ has integral kernel that is a $C^{\infty}$-function away from the diagonal $\omega=\omega^{\prime}$ and is bounded by $C(\lambda)\left|\omega-\omega^{\prime}\right|^{-d+\rho}$ as $\omega^{\prime} \rightarrow \omega$. 
3.2. Now, we consider a class of long-range magnetic potentials $A$ satisfying the following condition.

Assumption 3.2. $A \in C^{\infty}$ and

$$
A(x)=A^{(\infty)}(x)+A^{(\mathrm{reg})}(x),
$$

where $A^{(\infty)} \in C^{\infty}\left(\mathbb{R}^{d} \backslash\{0\}\right)$ is a homogeneous function of degree -1 satisfying the transversality condition

$$
\left\langle A^{(\infty)}(x), x\right\rangle=0, \quad x \neq 0,
$$

and, for all $\alpha$,

$$
\left|\partial^{\alpha} A^{(\mathrm{reg})}(x)\right|=O\left(|x|^{-\rho-|\alpha|}\right), \quad \rho>1 .
$$

It turns out that for such long-range potentials the usual wave operators exist. This fact was first observed in [6]; see also [10] for a different approach. Nevertheless, the structures of the SM are completely different in the short- and long-range cases. In the long-range case it is natural to regard the $\mathrm{SM}$ as a PsDO on the unit sphere. Its principal symbol can be expressed in terms of the circulation

$$
I(x, \xi)=\int_{-\infty}^{\infty}\left\langle A^{(\infty)}(x+t \xi), \xi\right\rangle d t, \quad x \neq 0, \quad \xi \neq 0, \quad\langle x, \xi\rangle=0,
$$

of the homogeneous part $A^{(\infty)}$ of $A$ over the line $x+t \xi, t \in \mathbb{R}$. Condition (3.4) implies that

$$
\left\langle A^{(\infty)}(x+t \xi), \xi\right\rangle=-t^{-1}\left\langle A^{(\infty)}(x+t \xi), x\right\rangle=O\left(|t|^{-2}\right)
$$

as $|t| \rightarrow \infty$, so that the integral (3.6) converges. Making the change of variables $t=$ $|x||\xi|^{-1} s$ in (3.6), we arrive at the identity

$$
I(x, \xi)=I(\hat{x}, \hat{\xi}), \quad \hat{x}=x /|x|, \quad \hat{\xi}=\xi /|\xi| ;
$$

i.e., the function $I(x, \xi)$ is homogeneous of degree 0 in both variables. Observe also that

$$
I(x,-\xi)=-I(x, \xi) .
$$

Theorem 3.3. Let Assumption 3.2 be satisfied, and let $S_{0}$ be the $\mathrm{PsDO}$ of class $\mathcal{S}^{0}$ with the principal symbol

$$
p(\omega, z)=\eta(z) \exp (i I(-z, \omega)), \quad \omega \in \mathbb{S}^{d-1}, \quad z \in \mathbb{R}^{d},\langle\omega, z\rangle=0,
$$

where $I(x, \xi)$ is the integral (3.6), and $\eta \in C^{\infty}, \eta(z)=0$ near zero, $\eta(z)=1$ for $|z| \geq 1 / 2$. Then the wave operators (3.1) exist, and for every $p$ the corresponding SM admits the representation

$$
S(\lambda)=S_{0}+S_{p}(\lambda)+\tilde{S}_{p}(\lambda),
$$

where $S_{p}(\lambda)$ is a PsDO of class $\mathcal{S}^{-\nu}, \nu=\min \{\rho-1,1\}$, and the kernel of the operator $\tilde{S}_{p}(\lambda)$ is a $C^{p}$-function of $\omega, \omega^{\prime} \in \mathbb{S}^{d-1}$.

This result almost coincides with Theorem 5.2 of [16. On the other hand, it is quite a particular case of the general result of [15, where a complete description of the amplitude of the PsDO $S(\lambda)$ was obtained for all potentials satisfying condition (3.2) for some $\rho>0$. Theorem 3.3 is especially adapted to the magnetic potentials $A(x)$ arising naturally from magnetic fields. The operator $S_{0}$ can be viewed as the first Born approximation to the SM. Of course, the PsDO $S_{0}$ is not determined uniquely by its principal symbol (3.9), but the difference of two PsDOs with the same principal symbol can be included in the operator $S_{p}(\lambda)$. 
3.3. Now we discuss the behavior of the $\mathrm{SM}$ with respect to the gauge transformations defined by the formula

$$
\tilde{H}=e^{i \phi} H e^{-i \phi}=(i \nabla+\tilde{A}(x))^{2},
$$

where

$$
\tilde{A}(x)=A(x)+\operatorname{grad} \phi(x) .
$$

Of course, $\operatorname{curl} \tilde{A}(x)=\operatorname{curl} A(x)$. We are particularly interested in functions $\phi(x)$ that are asymptotically homogeneous of degree zero.

We find a relationship between the wave operators $W\left(H, H_{0}\right)$ and $W\left(\tilde{H}, H_{0}\right)$.

Proposition 3.4. Suppose that the wave operators $W_{ \pm}\left(H, H_{0}\right)$ exist, and let a differentiable function $\phi$ be such that $\phi(x)=\phi_{0}(x)+\phi_{1}(x)$, where $\phi_{0}(x)=\phi_{0}(\hat{x})$ and $\phi_{1}(x)=o(1)$ as $|x| \rightarrow \infty$. Then the wave operators $W_{ \pm}\left(\tilde{H}, H_{0}\right)$ also exist and

$$
W_{ \pm}\left(\tilde{H}, H_{0}\right)=e^{i \phi(x)} W_{ \pm}\left(H, H_{0}\right) \mathcal{F}^{*} e^{-i \phi_{0}( \pm \xi)} \mathcal{F} .
$$

Proof. Since

$$
\left(\exp \left(-i H_{0} t\right) f\right)(x)=e^{i|x|^{2} /(4 t)}(2 i t)^{-d / 2} \hat{f}(x /(2 t))+o(1),
$$

we have

$$
\begin{aligned}
\left(e^{-i \phi} \exp \left(-i H_{0} t\right) f\right)(x) & =e^{i|x|^{2} /(4 t)}(2 i t)^{-d / 2} e^{-i \phi_{0}( \pm x /(2 t))} \hat{f}(x /(2 t))+o(1) \\
& =e^{i|x|^{2} /(4 t)}(2 i t)^{-d / 2} \hat{f}^{( \pm)}(x /(2 t))+o(1), \quad t \rightarrow \pm \infty,
\end{aligned}
$$

where $\hat{f}^{( \pm)}(\xi)=e^{-i \phi_{0}( \pm \xi)} \hat{f}(\xi)$, and the remainder $o(1)$ tends to 0 in $L_{2}\left(\mathbb{R}^{d}\right)$ as $t \rightarrow \pm \infty$. This is equivalent to the relation

$$
e^{-i \phi} \exp \left(-i H_{0} t\right) f=\exp \left(-i H_{0} t\right) f^{( \pm)}+o(1)
$$

which, in view of the definition (3.11), implies that

$$
\begin{aligned}
W_{ \pm}\left(\tilde{H}, H_{0}\right) f & =\lim _{t \rightarrow \pm \infty} e^{i \tilde{H} t} e^{-i H_{0} t} f=\lim _{t \rightarrow \pm \infty} e^{i \phi} e^{i H t} e^{-i \phi} e^{-i H_{0} t} f \\
& =\lim _{t \rightarrow \pm \infty} e^{i \phi} e^{i H t} e^{-i H_{0} t} f^{( \pm)}=e^{i \phi} W_{ \pm}\left(H, H_{0}\right) f^{( \pm)} .
\end{aligned}
$$

This proves (3.13).

As an immediate consequence of Proposition [3.4 we obtain a relationship between the scattering operators and matrices.

Proposition 3.5. Under the assumptions of Proposition 3.4, the scattering operators are related by the equation

$$
\mathcal{F} \mathbf{S}\left(\tilde{H}, H_{0}\right) \mathcal{F}^{*}=e^{i \phi_{0}(\xi)} \mathcal{F} \mathbf{S}\left(H, H_{0}\right) \mathcal{F}^{*} e^{-i \phi_{0}(-\xi)} .
$$

For all $\lambda>0$, the corresponding $\mathrm{SMs}, S(\lambda)=S\left(H, H_{0} ; \lambda\right)$ and $\tilde{S}(\lambda)=S\left(\tilde{H}, H_{0} ; \lambda\right)$, satisfy the relation

$$
\tilde{S}(\lambda)=e^{i \phi_{0}(\omega)} S(\lambda) e^{-i \phi_{0}(-\omega)},
$$

or, in terms of the scattering amplitudes,

$$
\tilde{s}\left(\omega, \omega^{\prime} ; \lambda\right)=e^{i \phi_{0}(\omega)-i \phi_{0}\left(-\omega^{\prime}\right)} s\left(\omega, \omega^{\prime} ; \lambda\right) .
$$


We emphasize that relations (3.14)-(3.16) for the scattering operators and matrices (but not (3.13) for the wave operators) depend only on the asymptotics $\phi_{0}$ of the function $\phi$. Apparently, for the first time, formulas (3.13) and (3.14) appeared in the paper [11] in the case where $d=2$ and under some assumptions on $A$. Actually, these formulas do not require any assumptions at all.

Proposition 3.5 shows that, in general, the SM is not the identity operator for the zero magnetic field.

Example 3.6. Let

$$
A(x)=\operatorname{grad} \phi(x),
$$

where $\phi(x)$ is as in Proposition 3.4. Then the wave operators $W_{ \pm}\left(H, H_{0}\right)$ exist and

$$
\begin{aligned}
W_{ \pm}\left(H, H_{0}\right) & =e^{i \phi(x)} \mathcal{F}^{*} e^{-i \phi_{0}( \pm \xi)} \mathcal{F}, \\
\mathbf{S}\left(H, H_{0}\right) & =\mathcal{F}^{*} e^{i \phi_{0}(\xi)-i \phi_{0}(-\xi)} \mathcal{F} .
\end{aligned}
$$

The corresponding SM is independent of $\lambda$ and

$$
S\left(H, H_{0}\right)=e^{i \phi_{0}(\omega)-i \phi_{0}(-\omega)} .
$$

Relations (3.15) or (3.16) show that the SM is not determined by the magnetic field $B(x)=\operatorname{curl} A(x)$ only, although we have an explicit formula that connects the SM in different gauges. This seems to contradict the following mental experiment. Suppose that a quantum particle interacts with a magnetic field. Note that it is exactly a field, not a potential, which can be created by our hands. However, to calculate the SM theoretically, we must introduce a magnetic potential and then solve the Schrödinger equation. Thus, the SM depends on a potential, and we can think that the particle itself chooses a gauge convenient for it. There could be (at least) two possible explanations for this seeming contradiction. The first is that the scattering amplitude $s\left(\omega, \omega^{\prime} ; \lambda\right)$ cannot be measured experimentally, although this is widely believed to be possible. From this point of view, only the (differential) scattering cross section

$$
\Sigma_{\text {diff }}\left(\omega, \omega^{\prime}, \lambda\right)=(2 \pi)^{d-1} \lambda^{-(d-1) / 2}\left|s\left(\omega, \omega^{\prime} ; \lambda\right)\right|^{2}, \quad \omega \neq \omega^{\prime}
$$

( $\omega^{\prime}$ is the incident direction of a beam of particles and $\omega$ is the direction of observation) can be found experimentally, which is compatible with (3.16). Another point of view is that the experimental devices used for observation of a quantum particle are not harmless and fix some specific gauge.

On the other hand, for a given field, the SM is stable under the short-range perturbations of a potential. More precisely, the following statement is true.

Proposition 3.7. Suppose that the wave operators $W_{ \pm}\left(H, H_{0}\right)$ exist and that $\operatorname{curl} A(x)=$ $\operatorname{curl} \tilde{A}(x)$ and

$$
\tilde{A}(x)-A(x)=O\left(|x|^{-\rho}\right), \quad \rho>1,
$$

as $|x| \rightarrow \infty$. Then the wave operators $W_{ \pm}\left(\tilde{H}, H_{0}\right)$ also exist, and the scattering operators and matrices for the pairs $H_{0}, H$ and $H_{0}, \tilde{H}$ coincide.

Proof. By Propositions 3.4 and 3.5, it suffices to show that $A(x)$ and $\tilde{A}(x)$ are related to each other as in (3.12) with a function $\phi(x)$ that has a limit (independent of $\hat{x}$ ) as $|x| \rightarrow \infty$. We define $\phi(x)$ as the curvilinear integral

$$
\phi(x)=\int_{\Gamma_{x}}\langle\tilde{A}(y)-A(y), d y\rangle
$$

taken between 0 and a variable point $x$. By the Stokes theorem, this integral does not depend on the choice of $\Gamma_{x}$, which implies (3.12). Moreover, choosing $\Gamma_{x}$ as the line 
segment connecting 0 and $x=r \omega, \omega \in \mathbb{S}^{d-1}$, and using (3.18), we see that the limit of $\phi(r \omega)$ as $r \rightarrow \infty$ exists. It remains to show that this limit does not depend on $\omega \in \mathbb{S}^{d-1}$. Again by the Stokes theorem,

$$
\phi\left(r \omega_{2}\right)-\phi\left(r \omega_{1}\right)=\int_{\mathbb{S}_{r}\left(\omega_{1}, \omega_{2}\right)}\langle\tilde{A}(y)-A(y), d y\rangle,
$$

where $\mathbb{S}_{r}\left(\omega_{1}, \omega_{2}\right)$ is the arc of the circle centered at the origin and passing through the points $r \omega_{1}$ and $r \omega_{2}$. Condition (3.18) implies that the integral (3.19) tends to 0 as $r \rightarrow \infty$.

\section{§4. Long-RAnge Aharonov-Bohm EFfeCt}

4.1. First we discuss the case where $d=2$. For a given magnetic field $B(x)=(0,0, B(x))$, $x \in \mathbb{R}^{2}$, the magnetic potential $A^{(\operatorname{tr})}(x)=\left(A_{1}^{(\operatorname{tr})}(x), A_{2}^{(\operatorname{tr})}(x), 0\right)$ satisfying equation (1.1) (or (1.2) ) and obeying the transversal gauge condition (1.9) can be constructed by the formulas

$$
A_{1}^{(\mathrm{tr})}(x)=-x_{2} \int_{0}^{1} B(s x) s d s, \quad A_{2}^{(\mathrm{tr})}(x)=x_{1} \int_{0}^{1} B(s x) s d s .
$$

If condition (1.10) is satisfied, then (4.1) implies that $A^{(\operatorname{tr})}(x)$ can be written as in (3.3), where $A^{(\infty)}$ is a homogeneous function of degree -1 and $A^{(\mathrm{reg})}(x)=O\left(|x|^{-\rho}\right)$ with $\rho=r-1$ as $|x| \rightarrow \infty$. Indeed, $A^{(\infty)}$ is given by the formula

$$
A^{(\infty)}(x)=a(\hat{x})\left(-x_{2}, x_{1}, 0\right)|x|^{-2}, \quad \hat{x}=x /|x|,
$$

where

$$
a(\hat{x})=\int_{0}^{\infty} B(s \hat{x}) s d s
$$

is a function on the unit circle, and

$$
A^{(\mathrm{reg})}(x)=|x|^{-2}\left(x_{2},-x_{1}\right) \int_{|x|}^{\infty} B(s \hat{x}) s d s .
$$

Moreover, if $B \in C^{\infty}\left(\mathbb{R}^{d}\right)$ and for all $\alpha$ we have

$$
\left|\partial^{\alpha} B(x)\right| \leq C_{\alpha}(1+|x|)^{-r-|\alpha|}, \quad r>2,
$$

then $A^{(\operatorname{tr})} \in C^{\infty}\left(\mathbb{R}^{d}\right), a \in C^{\infty}(\mathbb{S})$, and estimates (3.5) are valid for all $\alpha$.

Since $\operatorname{curl} A^{(\mathrm{tr})}(x)=O\left(|x|^{-r}\right)$ and $\operatorname{curl} A^{(\mathrm{reg})}(x)=O\left(|x|^{-r}\right)$, the representation (3.3) shows that $\operatorname{curl} A^{(\infty)}(x)=O\left(|x|^{-r}\right)$ with $r>2$. On the other hand, $\operatorname{curl} A^{(\infty)}(x)$ is a homogeneous function of degree -2 , whence, necessarily,

$$
\operatorname{curl} A^{(\infty)}(x)=0, \quad x \neq 0 .
$$

The same arguments (or (4.2) ) show that the transversality condition (3.4) is satisfied. Thus, the potential $A^{(\operatorname{tr})}(x)$ satisfies Assumption 3.2

By (1.3) and (4.2), the total magnetic flux is equal to

$$
\Phi=\int_{\mathbb{S}} a(\psi) d \psi
$$

We recall that $\omega^{( \pm)}$is obtained from $\omega \in \mathbb{S}$ by rotation by an angle of $\pm \pi / 2$ in the positive (counterclockwise) direction. Set

$$
f(\omega)=\int_{\mathbb{S}\left(\omega^{(-)}, \omega^{(+)}\right)} a(\psi) d \psi, \quad \omega \in \mathbb{S},
$$


where the integral is taken in the positive direction over the semicircle between the points $\omega^{(-)}$and $\omega^{(+)}$. Then

$$
f(\omega)+f(-\omega)=\Phi
$$

for any $\omega \in \mathbb{S}$. Comparing formulas (4.3) and (4.7), we can express the function $f(\omega)$ in terms of the magnetic field:

$$
f(\omega)=\int_{\langle x, \omega\rangle \geq 0} B(x) d x .
$$

In its turn, the integral (3.6) can be expressed in terms of the function $f(\omega)$.

Lemma 4.1. For all $\omega \in \mathbb{S}^{d-1}$, we have

$$
I\left(\omega, \omega^{( \pm)}\right)= \pm f(\omega) .
$$

Proof. By (3.8), it suffices to consider the case of the upper (plus) sign. Since

$$
\left\langle\left(-\omega_{2}-t \omega_{2}^{(+)}, \omega_{1}+t \omega_{1}^{(+)}\right),\left(\omega_{1}^{(+)}, \omega_{2}^{(+)}\right)\right\rangle=\omega_{1} \omega_{2}^{(+)}-\omega_{2} \omega_{1}^{(+)}=1,
$$

for the potentials (4.2) we have

$$
I\left(\omega, \omega^{(+)}\right)=\int_{-\infty}^{\infty} a\left(\frac{\omega+t \omega^{(+)}}{\sqrt{t^{2}+1}}\right) \frac{d t}{t^{2}+1} .
$$

Making the change of variables $t=\tan \psi$, we arrive at (4.10).

Let $S^{(\operatorname{tr})}(\lambda)$ denote the SM corresponding to the potential $A^{(\operatorname{tr})}$. By Theorem 3.3 , the following two assertions are immediate consequences of Propositions 2.1 and 2.2 (see also Remark [2.3).

Theorem 4.2. Let $d=2$, and let condition (4.4) be satisfied. Define $f(\omega)$ by (4.9) and set $\gamma_{+}=\max f(\omega), \gamma_{-}=\min f(\omega)$. Then for all $\lambda>0$ we have

$$
\sigma_{\text {ess }}\left(S^{(\operatorname{tr})}(\lambda)\right)=\left[\exp \left(i \gamma_{-}\right), \exp \left(i \gamma_{+}\right)\right] \cup\left[\exp \left(-i \gamma_{+}\right), \exp \left(-i \gamma_{-}\right)\right]
$$

if $\gamma_{+}-\gamma_{-}<2 \pi$, and $\sigma_{\mathrm{ess}}\left(S^{(\operatorname{tr})}(\lambda)\right)$ covers the entire unit circle $\mathbb{T} \subset \mathbb{C}$ if $\gamma_{+}-\gamma_{-} \geq 2 \pi$.

Proof. We apply Proposition 2.1 to the PsDO $S_{0}$ with the principal symbol (3.9). By (4.10), in this case we have

$$
p\left(\omega, \omega^{( \pm)}\right)=e^{ \pm i f\left(\omega^{(\mp)}\right)} .
$$

Therefore, if $\gamma_{+}-\gamma_{-}<2 \pi$, then the ranges of the functions $p\left(\omega, \omega^{(+)}\right)$and $p\left(\omega, \omega^{(-)}\right)$ coincide with the first and second arcs in (4.11), respectively. If $\gamma_{+}-\gamma_{-} \geq 2 \pi$, then each of these ranges covers the entire unit circle. Thus, it remains to take into account the fact that, by (3.10), the essential spectra of the operators $S^{(\operatorname{tr})}(\lambda)$ and $S_{0}$ are the same.

Theorem 4.3. Under the assumptions of Theorem 4.2, set

$$
s_{0}\left(\omega, \omega^{\prime}\right)=e^{i\left(f\left(\omega^{(-)}\right)-f\left(\omega^{(+)}\right)\right) / 2}\left(\cos (\Phi / 2) \delta\left(\omega, \omega^{\prime}\right)+\pi^{-1} \sin (\Phi / 2) \text { P.V. } \frac{\operatorname{sgn}\left\{\omega, \omega^{\prime}\right\}}{\left|\omega-\omega^{\prime}\right|}\right) .
$$

Then, for an arbitrary $\lambda>0$,

$$
\left|s^{(\operatorname{tr})}\left(\omega, \omega^{\prime} ; \lambda\right)-s_{0}\left(\omega, \omega^{\prime}\right)\right| \leq C(\lambda)\left|\omega-\omega^{\prime}\right|^{-3+r_{0}} .
$$

Here $r_{0}=r$ if $r<3$, and $r_{0}$ is an arbitrary number smaller than 3 if $r \geq 3$. 
Proof. Now we apply Proposition 2.2 to the PsDO $S_{0}$ with the principal symbol (3.9). Comparing formulas (2.3) and (4.12), we see that, in the case under consideration,

$$
p^{(\mathrm{av})}(\omega)=2^{-1}\left(e^{i f\left(\omega^{(-)}\right)}+e^{-i f\left(\omega^{(+)}\right)}\right), \quad p^{(s)}(\omega)=(2 \pi i)^{-1}\left(e^{i f\left(\omega^{(-)}\right)}-e^{-i f\left(\omega^{(+)}\right)}\right) .
$$

Using identity (4.8), we find that formula (2.3) yields the expression (4.13) for the singular part of the kernel of the operator $S_{0}$. The "regular" part of this kernel is $O\left(\left|\omega-\omega^{\prime}\right|^{-\varepsilon}\right)$ as $\left|\omega-\omega^{\prime}\right| \rightarrow 0$ for any $\varepsilon>0$. The kernel of the operator $S_{p}(\lambda)$ in (3.10) satisfies the same estimate if $r \geq 3$, and it is $O\left(\left|\omega-\omega^{\prime}\right|^{-3+r}\right)$ if $r<3$.

Corollary 4.4. The diagonal singularity of the scattering cross section (3.17) is given by the formula

$$
\Sigma_{\text {diff }}\left(\omega, \omega^{\prime} ; \lambda\right)=2 \pi^{-1} \lambda^{-1 / 2} \sin ^{2}(\Phi / 2)\left|\omega-\omega^{\prime}\right|^{-2}+O\left(\left|\omega-\omega^{\prime}\right|^{-4+r_{0}}\right) .
$$

Thus, the singular part $S_{0}$ of the SM $S^{(\operatorname{tr})}(\lambda)$ is the integral operator in $L_{2}(\mathbb{S})$ with the kernel (4.13). Up to a phase factor, this operator is determined by the magnetic flux $\Phi$ only (and does not depend on $\lambda$ ). We see that, in dimension two, even for compactly supported magnetic fields with $\Phi \notin 2 \pi \mathbb{Z}$, the SM involves a singular integral operator, and that the forward singularity (4.14) of the scattering cross section is stronger than for the short-range magnetic potentials, where it is $O\left(\left|\omega-\omega^{\prime}\right|^{-4+2 \rho}\right)$. On the contrary, if $\Phi \in 2 \pi \mathbb{Z}$, then, by (4.8) and (4.13), the operator $S_{0}$ acts as multiplication by the function $e^{i f\left(\omega^{(-)}\right)}$. As we shall see in the next section, this situation is typical in the case of dimensions $d \geq 3$. Note also that if $B(x)$ is an even function, that is, $B(x)=B(-x)$, then, again by (4.8), $f(\omega)=\Phi / 2$ for all $\omega \in \mathbb{S}$, so that the first factor on the right-hand side of (4.13) equals 1 . In this case, $\sigma_{\text {ess }}(S(\lambda))$ consists of the two points $e^{i \Phi / 2}$ and $e^{-i \Phi / 2}$. Of course, if $a(\omega)=-\alpha$, then formula (4.13) coincides, up to smooth terms, with formula (1.6), provided that the natural parametrization of the unit circle $\mathbb{S}$ by the points $\theta \in[0,2 \pi)$ is used.

As a specific example, consider the field

$$
B(x)=B_{0}(r)+B_{1}(r)\langle q, \hat{x}\rangle, \quad r=|x|,
$$

where $B_{0}$ and $B_{1}$ are $C^{\infty}$-functions with compact support and $q \in \mathbb{R}^{2}$ is a fixed vector. Formula (4.3) shows that in this case,

$$
a(\hat{x})=-\alpha+\langle p, \hat{x}\rangle, \quad \alpha \in \mathbb{R}, \quad p \in \mathbb{R}^{2},
$$

where

$$
\alpha=-\int_{0}^{\infty} B_{0}(r) r d r, \quad p=q \int_{0}^{\infty} B_{1}(r) r d r .
$$

Clearly, $\Phi=-2 \pi \alpha$. We calculate the function (4.7). For an arbitrary $\omega \in \mathbb{S}$, let $\varphi$ be the angle between $\omega$ and $p$, and let $\theta$ be the angle between $\hat{x}$ and $p$. Then $a(\hat{x})=-\alpha+|p| \cos \theta$ and

$$
f(\omega)=-\pi \alpha+|p| \int_{\varphi-\pi / 2}^{\varphi+\pi / 2} \cos \theta d \theta=-\pi \alpha+2|p| \cos \varphi=-\pi \alpha+2\langle p, \omega\rangle .
$$

Therefore, the conclusion of Theorem 4.2 is true with $\gamma_{+}=-\pi \alpha+2|p|$ and $\gamma_{-}=-\pi \alpha-$ $2|p|$. In particular, if $2|p| \geq \pi$, then $\sigma_{\text {ess }}\left(S^{(\operatorname{tr})}(\lambda)\right)=\mathbb{T}$. On the contrary, $\sigma_{\text {ess }}\left(S^{(\operatorname{tr})}(\lambda)\right)$ consists of the two points $\exp (\pi i \alpha)$ and $\exp (-\pi i \alpha)$ if $p=0$. The phase factor in (4.13) equals $\exp \left(2 i\left\langle p, \omega^{(-)}\right\rangle\right)$.

Actually, the results stated above do not require that the potential satisfy the transversality condition (1.9). The next result follows from Theorem 3.3 combined with Propositions 2.1 and 2.2 . 
Theorem 4.5. Suppose that $A \in C^{\infty}\left(\mathbb{R}^{2}\right)$ admits a representation (3.3), where $A^{(\infty)}$ is the function (4.2) with $a \in C^{\infty}(\mathbb{S})$, and $A^{(\mathrm{reg})}$ satisfies estimates (3.5). Let $f$ be the function (4.7). Then all conclusions of Theorems 4.2 and 4.3 remain true for the SM corresponding to the potential $A$.

We discuss this result from the viewpoint of gauge transformations. Let two potentials $A(x)$ and $\tilde{A}(x)$ satisfy the assumptions of Theorem 4.5 and let $\Phi$ and $\tilde{\Phi}$ be the corresponding magnetic fluxes. If they are related to each other as in (3.12) with $\phi(x)$ satisfying the conditions of Proposition 3.4, then $\tilde{\Phi}=\Phi$ and

$$
\tilde{a}(\omega)=a(\omega)+\phi_{0}^{\prime}(\omega)
$$

whence, by (4.7),

$$
\tilde{f}\left(\omega^{(-)}\right)-\tilde{f}\left(\omega^{(+)}\right)=f\left(\omega^{(-)}\right)-f\left(\omega^{(+)}\right)+2\left(\phi_{0}(\omega)-\phi_{0}(-\omega)\right) .
$$

Relation (4.13) implies that the singular parts of the corresponding SM satisfy the identity

$$
\tilde{s}_{0}\left(\omega, \omega^{\prime}\right)=e^{i \phi_{0}(\omega)-i \phi_{0}(-\omega)} s_{0}\left(\omega, \omega^{\prime}\right),
$$

which agrees with the exact formula (3.16) for scattering amplitudes.

Conversely, if $\tilde{\Phi}=\Phi$, then the function

$$
\phi_{0}(\omega)=\int_{\mathbb{S}\left(\omega_{0}, \omega\right)}(\tilde{a}(\psi)-a(\psi)) d \psi
$$

(the point $\omega_{0} \in \mathbb{S}$ is arbitrary but fixed) is well defined on the unit circle and (4.15) is satisfied. Set $\phi(x)=\eta(|x|) \phi_{0}(\hat{x})$, where $\eta \in C^{\infty}, \eta(r)=0$ in a neighborhood of zero, and $\eta(r)=1$ for large $r$. From (4.15) we see that (3.12) is fulfilled up to a term $A_{\mathrm{sr}}(x)$ satisfying estimates (3.5), that is, $\tilde{A}=A+\operatorname{grad} \phi+A_{\mathrm{sr}}$. Therefore, the SMs $S(\lambda)$ and $\tilde{S}(\lambda)$ for the Schrödinger operators with the magnetic potentials $A$ and $\tilde{A}-A_{\mathrm{sr}}$ satisfy (3.15), so that their singular parts satisfy (4.16). This implies that if (4.13) is fulfilled for $\tilde{A}-A_{\mathrm{sr}}$, then so it is for $A$. Thus, for a given $\Phi$, it suffices to prove Theorem 4.3 only for one function $a$ satisfying (4.6) (but for all short-range terms $A^{(\mathrm{reg})}$ ). We can choose $a(\omega)=(2 \pi)^{-1} \Phi$, which reduces the proof of Theorem 4.3 to the case of a constant function $a$. In particular, if $\Phi=0$, then the problem reduces to the short-range case. The same can be said about Theorem 4.2 for even functions $a(\omega)$ only. Then the function (4.17) is also even, so that, by (3.15), the $\mathrm{SMs} \tilde{S}(\lambda)$ and $S(\lambda)$ are unitarily equivalent.

4.2. Here we consider arbitrary magnetic potentials $A(x), x \in \mathbb{R}^{3}$, with Coulomb decay at infinity and satisfying, at least asymptotically, the transversal gauge condition. For such potentials, in general, the magnetic field $B(x)=\operatorname{curl} A(x)$ decays as $|x|^{-2}$ at infinity, so that the assumption (1.10) is not satisfied. We shall show that in this case the SM involves a singular integral operator and, hence, the long-range $\mathrm{AB}$ effect occurs.

The next two results extend Theorems 4.2 and 4.3 to the case where $d=3$. Again, they follow from Theorem 3.3 and Propositions 2.1 and 2.2 applied to the PsDO with the symbol (3.9).

Theorem 4.6. Suppose that $d=3$ and that Assumption 3.2 is satisfied. Let $I(x, \xi)$ be as in (3.6). Then $\sigma_{\mathrm{ess}}(S(\lambda))$ coincides with the range of the function $I(\psi, \omega)$ for all $\psi, \omega \in \mathbb{S}^{2}$ such that $\langle\psi, \omega\rangle=0$.

Theorem 4.7. Under the assumptions of Theorem 4.6, define the functions

$$
\begin{aligned}
p^{(\mathrm{av})}(\omega) & =(2 \pi)^{-1} \int_{\mathbb{S}_{\omega}} \exp (i I(\psi, \omega)) d \psi, \quad \mathbb{S}_{\omega}=\mathbb{S}^{2} \cap \Pi_{\omega}, \\
q(\omega, \tau) & =-(2 \pi)^{-2} \int_{\mathbb{S}_{\omega}}\left(\exp (i I(\psi, \omega))-p^{(\mathrm{av})}(\omega)\right)(\langle\psi, \tau\rangle-i 0)^{-2} d \psi,
\end{aligned}
$$


and set

$$
s_{0}\left(\omega, \omega^{\prime}\right)=p^{(\mathrm{av})}(\omega) \delta\left(\omega, \omega^{\prime}\right)+\text { P.V. } q\left(\omega, \omega^{\prime}-\omega\right) .
$$

Then for an arbitrary $\lambda>0$ the scattering amplitude satisfies the estimate

$$
\left|s\left(\omega, \omega^{\prime} ; \lambda\right)-s_{0}\left(\omega, \omega^{\prime}\right)\right| \leq C(\lambda)\left|\omega-\omega^{\prime}\right|^{-3+\rho_{0}},
$$

where $\rho_{0}=\rho$ if $\rho \in(1,2)$ and $\rho_{0}=2$ if $\rho \geq 2$.

Corollary 4.8. If $\omega \neq \omega^{\prime}$ but $\omega-\omega^{\prime} \rightarrow 0$, then

$$
s\left(\omega, \omega^{\prime} ; \lambda\right)=q\left(\omega, \omega^{\prime}-\omega\right)+O\left(\left|\omega-\omega^{\prime}\right|^{-3+\rho_{0}}\right) .
$$

Corollary 4.9. If $\omega \rightarrow \omega^{\prime}$, then

$$
\Sigma_{\text {diff }}\left(\omega, \omega^{\prime} ; \lambda\right)=(2 \pi)^{2} \lambda^{-1}\left|q\left(\omega, \omega^{\prime}-\omega\right)\right|^{2}+O\left(\left|\omega-\omega^{\prime}\right|^{-5+\rho_{0}}\right) .
$$

Note that here the order of singularity $\left|\omega-\omega^{\prime}\right|^{-4}$ is the same as for the electric Coulomb potentials. We emphasize also that the singular part (4.20) of the SM does not depend on $\lambda$.

Formula (4.19) shows that if the function $I(x, \omega)$ does not depend on $x$, then $q(\omega, \tau)=$ 0 , so that the singular integral operator in (4.20) disappears. In the next section we shall see that this situation really occurs if the magnetic field satisfies condition (1.10).

Nevertheless, $q(\omega, \tau)$ is nontrivial in the general case. We consider (see [16] for the details) two specific examples of potentials $A^{(\infty)}(x)$ homogeneous of degree -1 and satisfying the transversality condition (3.4). The corresponding fields curl $A(x)$ decay only as $|x|^{-2}$ at infinity.

The first of these potentials is defined by

$$
A^{(\infty)}(x)=|x|^{-3}\left(\alpha_{1} x_{2} x_{3}, \alpha_{2} x_{3} x_{1}, \alpha_{3} x_{1} x_{2}\right), \quad x=\left(x_{1}, x_{2}, x_{3}\right) \in \mathbb{R}^{3},
$$

where the $\alpha_{j}$ are constants and $\alpha_{1}+\alpha_{2}+\alpha_{3}=0$. An easy calculation shows that the function (3.6) is equal to

$$
I(x, \omega)=2|x|^{-2}\left(\alpha_{1} \omega_{1} x_{2} x_{3}+\alpha_{2} \omega_{2} x_{3} x_{1}+\alpha_{3} \omega_{3} x_{1} x_{2}\right) .
$$

Since this function depends on $x$, it cannot be expected that $q(\omega, \tau)=0$.

Actually, the functions $p^{(\mathrm{av})}(\omega)$ and $q(\omega, \tau)$ can be calculated explicitly. For any $\omega=\left(\omega_{1}, \omega_{2}, \omega_{3}\right)$, the coordinates of an arbitrary point $x=\left(x_{1}, x_{2}, x_{3}\right) \in \mathbb{S}_{\omega}=\mathbb{S}^{2} \cap \Pi_{\omega}$ can be written, for some $\theta \in[0,2 \pi)$, as

$$
\left\{\begin{array}{l}
x_{1}=-\left(\omega_{1}^{2}+\omega_{2}^{2}\right)^{-1 / 2}\left(\omega_{2} \cos \theta+\omega_{1} \omega_{3} \sin \theta\right) \\
x_{2}=\left(\omega_{1}^{2}+\omega_{2}^{2}\right)^{-1 / 2}\left(\omega_{1} \cos \theta-\omega_{2} \omega_{3} \sin \theta\right) \\
x_{3}=\left(\omega_{1}^{2}+\omega_{2}^{2}\right)^{1 / 2} \sin \theta
\end{array}\right.
$$

We set

$$
\mathcal{A}(\omega)=\left(\omega_{1}^{2}+\omega_{2}^{2}\right)^{-1}\left(4 \alpha_{3}^{2} \omega_{1}^{2} \omega_{2}^{2} \omega_{3}^{2}+\left(\alpha_{1}\left(\omega_{1}^{2}-\omega_{2}^{2} \omega_{3}^{2}\right)-\alpha_{2}\left(\omega_{2}^{2}-\omega_{1}^{2} \omega_{3}^{2}\right)\right)^{2}\right)^{1 / 2}
$$

and define the angle $\theta_{0}(\omega)$ by the formulas

$$
\left\{\begin{array}{l}
\sin \theta_{0}(\omega)=-2 \alpha_{3} \omega_{1} \omega_{2} \omega_{3}\left(\omega_{1}^{2}+\omega_{2}^{2}\right)^{-1} \mathcal{A}(\omega)^{-1} \\
\cos \theta_{0}(\omega)=\left(\alpha_{1}\left(\omega_{1}^{2}-\omega_{2}^{2} \omega_{3}^{2}\right)-\alpha_{2}\left(\omega_{2}^{2}-\omega_{1}^{2} \omega_{3}^{2}\right)\right)\left(\omega_{1}^{2}+\omega_{2}^{2}\right)^{-1} \mathcal{A}(\omega)^{-1} .
\end{array}\right.
$$

Then

$$
p^{(\mathrm{av})}(\omega)=(2 \pi)^{-1} \int_{0}^{2 \pi} \cos (\mathcal{A}(\omega) \sin \theta) d \theta
$$


and

$$
q(\omega, \tau)=-(2 \pi)^{-2} \int_{0}^{2 \pi}\left(e^{i \mathcal{A}(\omega) \sin \left(2 \theta+\theta_{0}(\omega)\right)}-p^{(\mathrm{av})}(\omega)\right)(\langle x(\theta), \tau\rangle-i 0)^{-2} d \theta,
$$

where $x(\theta)$ is defined by formulas (4.21).

As another example, we choose a modification of the $\mathrm{AB}$ potential

$$
A^{(\infty)}(x)=-\alpha|x|^{-2}\left(-x_{2}, x_{1}, 0\right) .
$$

In this case,

$$
I(x, \omega)=\pi \alpha|x|^{-1}\left(\omega_{1} x_{2}-\omega_{2} x_{1}\right)=\pi \alpha\left(1-\omega_{3}^{2}\right)^{1 / 2} \cos \theta
$$

if $x, \omega$, and $\theta$ are related as in (4.21). Plugging this in (4.18) and (4.19), we obtain explicit representations for the functions $p^{(\mathrm{av})}(\omega)=p^{(\mathrm{av})}\left(\omega_{3}\right)$ and $q(\omega, \tau)=q\left(\omega_{3}, \tau\right)$ :

$$
\begin{aligned}
p^{(\mathrm{av})}\left(\omega_{3}\right) & =(2 \pi)^{-1} \int_{0}^{2 \pi} \cos \left(\pi \alpha\left(1-\omega_{3}^{2}\right)^{1 / 2} \cos \theta\right) d \theta, \\
q\left(\omega_{3}, \tau\right) & =-(2 \pi)^{-2} \int_{0}^{2 \pi}\left(e^{i \pi \alpha\left(1-\omega_{3}^{2}\right)^{1 / 2} \cos \theta}-p^{(\mathrm{av})}(\omega)\right)(\langle x(\theta), \tau\rangle-i 0)^{-2} d \theta .
\end{aligned}
$$

In both of these examples the SM involves a singular integral operator, and hence, the long-range $\mathrm{AB}$ effect occurs.

\section{§5. There is no LOng-Range Aharonov-Bohm effect in Dimension three}

5.1. In this section we suppose that the dimension $d$ is 3 . Our results remain true for all $d \geq 3$, but not for $d=2$. Let $B(x)=\left(B_{1}(x), B_{2}(x), B_{3}(x)\right)$ be a magnetic field such that $\operatorname{div} B(x)=0$. We recall that a magnetic potential $A^{(\operatorname{tr})}(x)=\left(A_{1}^{(\operatorname{tr})}(x), A_{2}^{(\operatorname{tr})}(x), A_{3}^{(\operatorname{tr})}(x)\right)$ satisfying equation (1.1) and the transversal gauge condition (1.9) is constructed by the formula

$$
A_{1}^{(\mathrm{tr})}(x)=\int_{0}^{1}\left(B_{2}(s x) x_{3}-B_{3}(s x) x_{2}\right) s d s .
$$

The expressions for the components $A_{2}^{(\operatorname{tr})}(x)$ and $A_{3}^{(\operatorname{tr})}(x)$ are obtained by cyclic permutations of the indices in (5.1). If estimate (1.10) is satisfied, then $A^{(\operatorname{tr})}(x)$ admits a representation (3.3), where

$$
\begin{gathered}
A_{1}^{(\infty)}(x)=|x|^{-2} \int_{0}^{\infty}\left(B_{2}(s \hat{x}) x_{3}-B_{3}(s \hat{x}) x_{2}\right) s d s, \\
A_{1}^{(\mathrm{reg})}(x)=-|x|^{-2} \int_{|x|}^{\infty}\left(B_{2}(s \hat{x}) x_{3}-B_{3}(s \hat{x}) x_{2}\right) s d s .
\end{gathered}
$$

Thus, $A^{(\infty)}(x)$ is a homogeneous function of degree -1 , and $A^{(\mathrm{reg})}(x)=O\left(|x|^{-\rho}\right)$ with $\rho=r-1>1$ as $|x| \rightarrow \infty$. As in the two-dimensional case (see Subsection 4.1), we check that $A^{(\infty)}(x)$ satisfies equations (3.4) and (4.5).

Given a magnetic field $B(x)$ obeying condition (1.10), we now construct a magnetic potential $A(x)$ satisfying equation (1.1) and estimate (1.7). We start with the magnetic potential $A^{(\operatorname{tr})}$ in the transversal gauge. Let $A^{(\infty)}$ be the function (5.2). We introduce the function $U(x)$ for $x \neq 0$ as the curvilinear integral

$$
U(x)=\int_{\Gamma_{x_{0}, x}}\left\langle A^{(\infty)}(y), d y\right\rangle
$$

taken between some fixed point $x_{0} \neq 0$ and a variable point $x$. It is assumed that $0 \notin \Gamma_{x_{0}, x}$, and then, by (4.5) and the Stokes theorem, $U(x)$ does not depend on the 
choice of a contour $\Gamma_{x_{0}, x}$. We have used the fact that the set $\mathbb{R}^{3} \backslash\{0\}$ (and $\mathbb{R}^{d} \backslash\{0\}$ for all $d \geq 3$ ) is simply connected. Clearly,

$$
A^{(\infty)}(x)=\operatorname{grad} U(x)
$$

Moreover, the function $U(x)$ is homogeneous of degree 0. Indeed, if $x_{2}=\gamma x_{1}, \gamma>1$, then we can choose $\Gamma_{x_{0}, x_{2}}=\Gamma_{x_{0}, x_{1}} \cup\left(x_{1}, x_{2}\right)$, where $\left(x_{1}, x_{2}\right)$ is the line segment connecting $x_{1}$ and $x_{2}$. If $y \in\left(x_{1}, x_{2}\right)$, then $\left\langle A^{(\infty)}(y), d y\right\rangle=0$ by (3.4). Consequently, $U\left(x_{1}\right)=U\left(x_{2}\right)$. We use the definition (5.4) away from some neighborhood of the point $x=0$ and extend $U(x)$ to $\mathbb{R}^{3}$ as a differentiable function. For instance, we can choose numbers $R_{2}>R_{1}>0$ and a function $\eta \in C^{\infty}\left(\mathbb{R}^{3}\right)$ such that $\eta(x)=1$ for $|x| \geq R_{2}, \eta(x)=0$ for $|x| \leq R_{1}$ and then replace $U(x)$ with $\eta(x) U(x)$. Now we set

$$
\begin{aligned}
A(x) & =A^{(\operatorname{tr})}(x)-\operatorname{grad}(\eta(x) U(x)) \\
& =A^{(\mathrm{reg})}(x)+(1-\eta(x)) A^{(\infty)}(x)-U(x) \operatorname{grad} \eta(x),
\end{aligned}
$$

so that $A(x)=A^{(\mathrm{reg})}(x)$ for $|x| \geq R_{2}$ and $A(x)=A^{(\operatorname{tr})}(x)$ for $|x| \leq R_{1}$. Thus, we arrive at the following result.

Proposition 5.1. Suppose that $\operatorname{div} B(x)=0$ and that condition (1.10) is satisfied. Let $A(x)$ be the magnetic potential defined by formula (5.6) where $A^{(\infty)}(x), A^{(\mathrm{reg})}(x)$, and $U(x)$ are the functions (5.2), (5.3), and (5.4), respectively. Then $A(x)$ satisfies equation (1.1) and estimate (1.7). Moreover, $A(x)$ has compact support if $B(x)$ has compact support.

In the case of magnetic fields $B(x)$ with compact support, our construction is close to that of 3 . In the proof of Proposition 5.1 we could have started with a magnetic potential $A^{(c)}(x)$ satisfying the Coulomb gauge condition $\operatorname{div} A^{(c)}(x)=0$. However, this is less convenient.

Suppose that a magnetic field is localized in some ball $\mathbb{B}, \mathbb{B}^{\prime}$ is a slightly larger ball, and a direct interaction of quantum particles with this field is excluded by the Dirichlet boundary condition on $\partial \mathbb{B}^{\prime}$. Proposition 5.1 shows that we can choose a magnetic potential with support in $\mathbb{B}^{\prime}$, so that scattering is trivial in this case. On the other hand, if a magnetic field is supported on some torus $\mathbf{T}$ and the Dirichlet boundary condition is put on the boundary of a slightly larger torus $\mathbf{T}^{\prime}$, then the Stokes theorem does not allow us to find a potential supported on $\mathbf{T}^{\prime}$ (provided the magnetic flux through a section of $\mathbf{T}$ is not zero). Therefore, scattering is nontrivial in this case, although it is of a short-range nature.

Let $\mathcal{A}(B)$ denote the class of magnetic potentials satisfying equation (1.1) and estimate (1.7) for some $\rho>1$. This class is nonempty by Proposition 5.1. If $A \in \mathcal{A}(B)$, then, for an arbitrary function $\phi(x)$ such that $\operatorname{grad} \phi(x)=O\left(|x|^{-\rho}\right)$, the potential (3.12) also belongs to this class. By Proposition 3.7 the SM for the pair $H_{0}=-\Delta, H=(i \nabla+A(x))^{2}$ does not depend on the choice of $A \in \mathcal{A}(B)$ and, thus, is determined by the magnetic field $B(x)$ only. We say that this SM $S(\lambda)=S(\lambda ; B)$ is the SM for the field $B(x)$.

Comparing Propositions 3.1 and 5.1, we arrive at the following result.

Theorem 5.2. Suppose a magnetic field $B(x)$ is such that $\operatorname{div} B(x)=0$, condition (1.10) is satisfied, and $A$ is a magnetic potential $\mathcal{A}(B)$. Then the wave operators for the pair $H_{0}=-\Delta, H=(i \nabla+A(x))^{2}$ exist and are unitary, and the $S M S(\lambda)$ for the magnetic field $B(x)$ is a unitary operator for all $\lambda>0$. The operator $T(\lambda)=S(\lambda)-I$ is compact and belongs to the trace class if $r>4$. If $r>4+n, n=0,1,2, \ldots$, then $T(\lambda)$ is an integral operator with kernel of class $C^{n}\left(\mathbb{S}^{2} \times \mathbb{S}^{2}\right)$. If condition (4.4) is fulfilled for some $r \in(2,4)$ and all multi-indices $\alpha$, then the operator $T(\lambda)$ has an integral kernel that is 
a $C^{\infty}$-function away from the diagonal $\omega=\omega^{\prime}$ and is bounded by $C(\lambda)\left|\omega-\omega^{\prime}\right|^{-4+r}$ as $\omega^{\prime} \rightarrow \omega$.

Corollary 5.3. If estimate (1.10) is satisfied for $r>4$, then $\Sigma_{\mathrm{diff}}\left(\omega, \omega^{\prime} ; \lambda\right)$ is a bounded function of $\omega, \omega^{\prime} \in \mathbb{S}^{2}$. If condition (4.4) is satisfied for some $r \in(2,4)$ and all multiindices $\alpha$, then

$$
\Sigma_{\text {diff }}\left(\omega, \omega^{\prime} ; \lambda\right)=O\left(\left|\omega-\omega^{\prime}\right|^{-8+2 r}\right) \quad \text { as } \omega \rightarrow \omega^{\prime} .
$$

Using the first formula in (5.6) and applying Proposition 3.5 to the function $\phi(x)=$ $U(x)$, we can also describe the structure of the SM in the transversal gauge.

Proposition 5.4. Suppose that a magnetic field $B(x)$ satisfies the assumptions of Theorem 5.2. Let $S^{(\operatorname{tr})}(\lambda)$ be the $S M$ for the pair $H_{0}=-\Delta, H=\left(i \nabla+A^{(\operatorname{tr})}(x)\right)^{2}$. Set

$$
u(\omega)=U(\omega)-U(-\omega),
$$

where the function $U(x)$ is defined by formula (5.4). Let $S_{0}$ be the operator of multiplication by the function $\exp (i u(\omega))$. Then all the results of Theorem 5.2 concerning the operator $T(\lambda)$ are true for the operator $T^{(\operatorname{tr})}(\lambda)=S^{(\operatorname{tr})}(\lambda)-S_{0}$.

If a magnetic field $B(x)$ satisfies the assumption (4.4), then, as in the two-dimensional case (see Subsection 4.1), Proposition 5.4 can be deduced from Theorem 3.3 and Proposition 2.2. Such an approach was used in [8]. Indeed, if curl $A(x)=o\left(|x|^{-2}\right)$ as $|x| \rightarrow \infty$, then condition (4.5) is necessarily satisfied, so that the function (5.4) is well defined. In this case the function $I(x, \omega)$ does not depend on $x$ and is equal to $u(\omega)$. Actually, (5.5) implies that

$$
\begin{aligned}
I(x, \omega) & =\int_{-\infty}^{\infty}\langle\operatorname{grad} U(x+t \omega), \omega\rangle d t=\lim _{T \rightarrow \infty} \int_{-T}^{T} \frac{d}{d t} U(x+t \omega) d t \\
& =\lim _{T \rightarrow \infty}(U(x+T \omega)-U(x-T \omega))=U(\omega)-U(-\omega) .
\end{aligned}
$$

Therefore, the function (4.19) is zero, and the singular integral operator in (4.20) disappears.

In dimension 2, the above construction works if (and only if) the total magnetic flux $\Phi$ is zero. Indeed, in this case

$$
\int_{|x|=R}\left\langle A^{(\infty)}(x), d x\right\rangle=0
$$

for any $R>0$, so that the function (5.4) is well defined again. Then Proposition 5.1 for the potential (5.6) and Theorem 5.2 for the SM remain true. The only difference is that under the assumption (4.4) the integral kernel of the operator $T(\lambda)$ is $O\left(\left|\omega-\omega^{\prime}\right|^{-3+r}\right)$ as $\omega-\omega^{\prime} \rightarrow 0$.

5.2. As an example, in the space $\mathbb{R}^{3}$ we consider a toroidal solenoid $\mathbf{T}$ symmetric with respect to rotations around the $x_{3}$-axis (the latter does not intersect $\mathbf{T}$ ). Suppose (which looks quite realistic) that the magnetic field is given by the formula

$$
B\left(x_{1}, x_{2}, x_{3}\right)=-\alpha\left(x_{1}^{2}+x_{2}^{2}\right)^{-1}\left(-x_{2}, x_{1}, 0\right), \quad \alpha=\text { const },
$$

inside $\mathbf{T}$ and is zero outside. Then $\operatorname{div} B(x)=0$ and the current curl $B(x)$ is zero if $x \notin \partial \mathbf{T}$. Of course, Theorem 5.2 applies to this field; hence, $S(\lambda)-I$ is an integral operator with kernel of class $C^{\infty}\left(\mathbb{S}^{2} \times \mathbb{S}^{2}\right)$.

We illustrate our construction by this example. First, we construct the potential $A^{(\operatorname{tr})}(x)$ by formula (5.1). We assume that the section $\mathbf{S}$ of $\mathbf{T}$, say, by the half-plane $x_{2}=0, x_{1} \geq 0$ is strictly convex and has a smooth boundary $\partial \mathbf{S}$ but is not necessarily a disk. Let $L_{z}, z \in \mathbb{R}$, be the half-line consisting of the points $s\left(1+z^{2}\right)^{-1 / 2}(1,0, z)$ for 
all $s \in \mathbb{R}_{+}$. We denote by $z_{1}$ and $z_{2}$ the values of $z$ for which $L_{z}$ is tangent to $\partial \mathbf{S}$ and, for $z \in\left[z_{1}, z_{2}\right]$, denote by $\varkappa_{ \pm}(z), \varkappa_{+}(z) \geq \varkappa_{-}(z)$, the values of $s$ for which $L_{z}$ intersects S. For $x=\left(x_{1}, x_{2}, x_{3}\right)$, set $z=z(x)=x_{3}\left(x_{1}^{2}+x_{2}^{2}\right)^{-1 / 2}$. Taking the rotational symmetry into account, we see that $s x \in \mathbf{T}$ if and only if $s|x|\left(1+z^{2}\right)^{-1 / 2}(1,0, z) \in \mathbf{S}$ or $\varkappa_{-}(z) \leq$ $s|x| \leq \varkappa_{+}(z)$. Thus, the integral (5.1) equals zero (and hence $\left.A^{(\operatorname{tr})}(x)=0\right)$ if $z(x) \notin$ $\left(z_{1}, z_{2}\right)$, and this integral is actually taken over the set $[0,1] \cap\left[\varkappa_{-}(z)|x|^{-1}, \varkappa_{+}(z)|x|^{-1}\right]$ if $z(x) \in\left(z_{1}, z_{2}\right)$. Therefore, $A^{(\operatorname{tr})}(x)=0$ if $|x| \leq \varkappa_{-}(z)$,

$$
\begin{aligned}
A_{1}^{(\mathrm{tr})}(x) & =-\alpha x_{1} x_{3}\left(x_{1}^{2}+x_{2}^{2}\right)^{-1} \int_{\varkappa_{-}(z) /|x|}^{1} d s \\
& =-\alpha x_{1} x_{3}\left(x_{1}^{2}+x_{2}^{2}\right)^{-1}\left(1-\varkappa_{-}(z) /|x|\right)=: A_{1}^{(0)}(x)
\end{aligned}
$$

if $\varkappa_{-}(z) \leq|x| \leq \varkappa_{+}(z)$, and

$$
\begin{aligned}
A_{1}^{(\operatorname{tr})}(x) & =-\alpha x_{1} x_{3}\left(x_{1}^{2}+x_{2}^{2}\right)^{-1} \int_{\varkappa_{-}(z) /|x|}^{\varkappa_{+}(z) /|x|} d s \\
& =-\alpha x_{1} x_{3}\left(x_{1}^{2}+x_{2}^{2}\right)^{-1}|x|^{-1}\left(\varkappa_{+}(z)-\varkappa_{-}(z)\right)
\end{aligned}
$$

if $|x| \geq \varkappa_{+}(z)$. The components $A_{2}^{(\operatorname{tr})}(x)$ and $A_{3}^{(\operatorname{tr})}(x)$ can be found in a similar way. In particular,

$$
\begin{aligned}
& A_{1}^{(\infty)}(x)=x_{1} x_{3}\left(x_{1}^{2}+x_{2}^{2}\right)^{-1}|x|^{-1} g(z), \\
& A_{2}^{(\infty)}(x)=x_{2} x_{3}\left(x_{1}^{2}+x_{2}^{2}\right)^{-1}|x|^{-1} g(z), \\
& A_{3}^{(\infty)}(x)=-|x|^{-1} g(z),
\end{aligned}
$$

where

$$
g(z)=-\alpha\left(\varkappa_{+}(z)-\varkappa_{-}(z)\right) .
$$

Clearly, $g(z)$ is a continuous function, $\pm g(z)>0$ if $\mp \alpha>0$ for $z \in\left(z_{1}, z_{2}\right)$, and $g(z)=0$ for $z \notin\left(z_{1}, z_{2}\right)$.

Let $\mathbf{K}$ be the cone in $\mathbb{R}^{3}$ where $z(x) \in\left[z_{1}, z_{2}\right]$. Then $\mathbf{T} \subset \mathbf{K}$, and $\mathbf{T}$ and $\mathbf{K}$ are tangent to each other. The internal (external) part of $\mathbf{K} \backslash \mathbf{T}$ will be denoted by $\mathbf{K}_{\text {int }}\left(\mathbf{K}_{\text {ext }}\right)$. Of course, $A^{(\operatorname{tr})}(x)=0$ if $x \notin \mathbf{K}$. By (5.8) and (5.9), we have

$$
\begin{aligned}
& A^{(\operatorname{tr})}(x)=0, \quad x \in \mathbf{K}_{\mathrm{int}}, \\
& A^{(\operatorname{tr})}(x)=A^{(0)}(x), \quad x \in \mathbf{T}, \\
& A^{(\operatorname{tr})}(x)=A^{(\infty)}(x), \quad x \in \mathbf{K}_{\text {ext }} .
\end{aligned}
$$

Now, formula (3.3) for $A^{(\operatorname{tr})}(x)$ shows that

$$
\begin{array}{ll}
A^{(\mathrm{reg})}(x)=-A^{(\infty)}(x), & x \in \mathbf{K}_{\mathrm{int}}, \\
A^{(\mathrm{reg})}(x)=A^{(0)}(x)-A^{(\infty)}(x), & x \in \mathbf{T}, \\
A^{(\mathrm{reg})}(x)=0, & x \in \mathbf{K}_{\text {ext }} .
\end{array}
$$

Taking (5.10) into account, we see that a function $U(x)$ satisfying (5.5) can be constructed by the explicit formula

$$
U(x)=G\left(x_{3}\left(x_{1}^{2}+x_{2}^{2}\right)^{-1 / 2}\right),
$$

where

$$
G^{\prime}(z)=-g(z)\left(z^{2}+1\right)^{-1 / 2}
$$


In particular, $U(x)$ is a constant for $z(x) \notin\left(z_{1}, z_{2}\right)$. Since $U(x)$ is defined up to a constant, we can set $U(x)=0$ for $z(x) \leq z_{1}$. Then

$$
U(x)=U_{0}=-\int_{-\infty}^{\infty} g(t)\left(t^{2}+1\right)^{-1 / 2} d t
$$

for $z(x) \geq z_{2}$. It is easy to check that $-U_{0}$ is equal to the magnetic flux $\Phi_{s}$ through the section $\mathbf{S}$ of the solenoid $\mathbf{T}$. Indeed, let $\omega_{0}=(0,0,1),\left\langle x_{0}, \omega_{0}\right\rangle=0$, and let $\left|x_{0}\right|$ and $R$ be sufficiently large. By the Stokes theorem, $\Phi_{s}$ is equal to the circulation of the potential $A^{(\operatorname{tr})}(x)$ over the closed contour formed by the four intervals $\left(-R \omega_{0}, R \omega_{0}\right)$, $\left(R \omega_{0}, R \omega_{0}+x_{0}\right),\left(R \omega_{0}+x_{0},-R \omega_{0}+x_{0}\right)$, and $\left(-R \omega_{0}+x_{0},-R \omega_{0}\right)$. Observe that $A^{(\operatorname{tr})}(x) \neq$ 0 only on the interval $\left(R \omega_{0}+x_{0},-R \omega_{0}+x_{0}\right)$, where $A^{(\operatorname{tr})}(x)=A^{(\infty)}(x)$, so that

$$
\Phi_{s}=-\int_{-R}^{R}\left\langle A^{(\infty)}\left(x_{0}+t \omega_{0}\right), \omega_{0}\right\rangle d t .
$$

Passing to the limit as $R \rightarrow \infty$ and using (3.6), we see that $\Phi_{s}=-I\left(x_{0}, \omega_{0}\right)$. Consequently, by (5.7), we have

$$
\Phi_{s}=-U\left(\omega_{0}\right)=-U_{0} .
$$

Now suppose that the number $R_{2}$ in the definition of the cut-off function $\eta(x)$ is chosen in such a way that the ball $|x| \leq R_{2}$ does not intersect $\mathbf{T}$. First, let $x \notin \mathbf{K}$, so that $A^{(\operatorname{tr})}(x)=0$. Then the first formula in (5.6) shows that $A(x)=0$ if $z(x) \leq z_{1}$, and

$$
A(x)=-U_{0} \operatorname{grad} \eta(x), \quad x \notin \mathbf{K}, \quad z(x) \geq z_{2} .
$$

If $x \in \mathbf{K}$, then, by (5.11) and the second formula in (5.6),

$$
\begin{aligned}
A(x) & =0, & |x| & \leq R_{1}, \\
A(x) & =-\operatorname{grad}(\eta(x) U(x)), & R_{1} \leq|x| & \leq R_{2}, \\
A(x) & =A^{(\operatorname{reg})}(x), & |x| & \geq R_{2} .
\end{aligned}
$$

In particular, $A(x)=0$ if $x \in \mathbf{T}_{\text {ext }}$.

The function $g(z)$ can be calculated explicitly if $\mathbf{S}$ is a disk. Suppose that this disk has radius $r$, its center belongs to the $x_{3}$-axis, and the distance from the center to the $x_{3}$-axis is $l, l>r$. Then the equation of $\partial \mathbf{T}$ is

$$
\left(\left(x_{1}^{2}+x_{2}^{2}\right)^{1 / 2}-l\right)^{2}+x_{3}^{2}=r^{2} .
$$

Setting $x_{2}=0, x_{3}=z x_{1}$, we obtain an equation for $x_{1}=x_{1}(z)$. The roots of this equation give us the numbers $\left(1+z^{2}\right)^{-1 / 2} \varkappa_{ \pm}(z)$. Thus,

$$
\varkappa_{ \pm}(z)=\left(1+z^{2}\right)^{-1 / 2}\left(l \pm\left(r^{2}-\left(l^{2}-r^{2}\right) z^{2}\right)^{1 / 2}\right)
$$

whence

$$
g(z)=-2 \alpha\left(1+z^{2}\right)^{-1 / 2}\left(r^{2}-\left(l^{2}-r^{2}\right) z^{2}\right)^{1 / 2} .
$$

In particular, $-z_{1}=z_{2}=r\left(l^{2}-r^{2}\right)^{-1 / 2}$ for this function.

Returning to the general case, we emphasize that a potential $A(x)$ satisfying the conclusions of Proposition 5.1 is highly nonunique. Actually, the gradient of an arbitrary short-range function can be added to $A(x)$. For example, in the case of (5.15) a magnetic potential completely different from that constructed above can be found in the book 1 .

Finally, we calculate the SM $S^{(\operatorname{tr})}(\lambda)$. By Proposition 5.4 up to an integral operator with a $C^{\infty}$-kernel, the $\operatorname{SM} S^{(\operatorname{tr})}(\lambda)$ is the operator $S_{0}$ of multiplication by the function $\exp (i u(\omega))$, where, by (5.12) and (5.13),

$$
u(\omega)=q\left(\omega_{3}\left(1-\omega_{3}^{2}\right)^{-1 / 2}\right), \quad \omega=\left(\omega_{1}, \omega_{2}, \omega_{3}\right) \in \mathbb{S}^{2},
$$


and

$$
q(z)=G(z)-G(-z)=-\int_{-z}^{z} g(t)\left(t^{2}+1\right)^{-1 / 2} d t .
$$

Clearly, $q(-z)=-q(z), q(z)$ is a monotone increasing (decreasing) function if $\alpha>0$ $(\alpha<0)$, and $q(z)$ is a constant, $q(z)=q_{0}$, if $z \geq \max \left\{\left|z_{1}\right|,\left|z_{2}\right|\right\}$. Relation (5.14) implies that

$$
q_{0}=u\left(\omega_{0}\right)=U\left(\omega_{0}\right)-U\left(-\omega_{0}\right)=-\Phi_{s}
$$

Thus, $u(\omega)$ depends only on the coordinate $\omega_{3}$ and takes all the values between $-\left|\Phi_{s}\right|$ and $\left|\Phi_{s}\right|$. Therefore, $\sigma_{\text {ess }}\left(S^{(\operatorname{tr})}(\lambda)\right)$ coincides with the $\operatorname{arc}\left[e^{-i\left|\Phi_{s}\right|}, e^{i\left|\Phi_{s}\right|}\right]$ if $\left|\Phi_{s}\right|<\pi$, and covers the entire unit circle if $\left|\Phi_{s}\right| \geq \pi$.

\section{REFERENCES}

[1] G. N. Afanasiev, Topological effects in quantum mechanics, Fundamental Theories of Physics, vol. 107, Kluwer, Acad. Publ. Group, Dordrecht, 1999. MR1730630 (2001c:81098)

[2] Y. Aharonov and D. Bohm, Significance of electromagnetic potentials in the quantum theory, Phys. Rev. (2) 115 (1959), 485-491. MR0110458 (22:1336)

[3] R. Hempel and I. Herbst, Strong magnetic fields, Dirichlet boundaries, and spectral gaps, Comm. Math. Phys. 169 (1995), 237-259. MR1329195 (96a:81026)

[4] W. C. Henneberger, Some aspects of the Aharonov-Bohm effect, Phys. Rev. A (3) 22 (1980), 13831388. MR0587271(81j:81004)

[5] I. Herbst, On the connectedness structure of the Coulomb S-matrix, Comm. Math. Phys. 35 (1974), 181-191. MR0351325 (50:3814)

[6] M. Loss and B. Thaller, Scattering of particles by long-range magnetic fields, Ann. Physics 176 (1987), 159-180. MR0893482 (88f:81133)

[7] M. Peshkin and A. Tonomura, The Aharonov-Bohm effect, Lecture Notes in Phys., vol. 340, Springer-Verlag, Berlin, 1989. MR.1016149 (91c:81004)

[8] P. Roux, La matrice de diffusion pour l'opérateur de Schrödinger avec un potentiel électromagnétique à longue portée, Ph. D. thesis, Univ. de Rennes-1, 2001.

[9] P. Roux and D. R. Yafaev, On the mathematical theory of the Aharonov-Bohm effect, J. Phys. A 35 (2002), 7481-7492. MR:1945524(2003m:81085)

[10] _ The scattering matrix for the Schrödinger operator with a long-range electromagnetic potential, J. Math. Phys. 44 (2003), 2762-2786. MR1982788(2004i:81260)

[11] S. N. M. Ruijsenaars, The Aharonov-Bohm effect and scattering theory, Ann. Physics 146 (1983), 1-34. MR0701261 (85e:81117)

[12] M. A. Shubin, Pseudodifferential operators and spectral theory, "Nauka", Moscow, 1978; English transl., Springer-Verlag, Berlin, 1987. MR0509034 (80h:47057) MR0883081 (88c:47105)

[13] D. R. Yafaev, On the classical and quantum Coulomb scattering, J. Phys. A 30 (1997), 6981-6992. MR 1481360 (99b:81259)

[14] _ Scattering theory: Some old and new problems, Lecture Notes in Math., vol. 1735, SpringerVerlag, Berlin, 2000. MR,1774673 (2001j:81248)

[15] _ High-energy and smoothness asymptotic expansion of the scattering amplitude, J. Funct. Anal. 202 (2003), 526-570. MR1990537 (2004f:35134)

[16] _ Scattering matrix for magnetic potentials with Coulomb decay at infinity, Integral Equations Operator Theory 47 (2003), 217-249. MR2002666 (2004f:47018)

IRMaR, Université Rennes-I, Campus Beaulieu, 35042 Rennes Cedex, France

E-mail address: yafaev@univ-rennes1.fr

Received 20/JAN/2005

Translated by THE AUTHOR 